\title{
Oral Health and Dental Veneers: Clinical Tips
}

\author{
Asra Sabir Hussain* \\ Post graduate student, University of Edinburgh, UK \\ *Corresponding author: Asra Sabir Hussain, BDS Post graduate student, University of Edinburgh, United Kingdom \\ To Cite This Article: Asra Sabir Hussain. Oral Health and Dental Veneers: Clinical Tips. Am J Biomed Sci \& Res. 2019 - 2(3). AJBSR.MS.ID.000584. \\ DOI: $10.34297 / A J B S R .2019 .02 .000584$
}

Received: March 26, 2019 | Published: April 08, 2019

\section{Introduction}

Porcelain Veneers are widely used nowadays to recreate the natural cosmetic appearance of teeth. It also provides strength and resilience in relevance to natural tooth enamel. Therefore, to enhance the clinical predictability of Porcelain veneers, Patients are commonly advised to follow routine oral health care recommendations by the Dental Professionals [1].

\section{Overview}

Maintenance of Porcelain veneers doesn't require any special oral health care. It's few clinical tips that are essential key to sustain veneers for a long time [2].

\section{Oral hygiene Practices}

Routine good oral hygiene practices are commonly advised by the dental professionals. Patients should treat veneers in the same manner likewise natural teeth. Routine oral hygiene practices include brushing (two minutes, twice daily) with proper technique, flossing and rinsing after meals with an antiseptic mouthwash [3]. Moreover, the use of non-abrasive fluoride toothpaste is typically recommended by the Dentists [1]. A soft toothbrush is required for brushing as it doesn't wear away the shine and polish of porcelain veneers.

Proper oral hygiene practices won't just clean and protect veneers, it also aids in minimizing the risk of developing gum diseases, thus it ultimately prevents gingivitis and gingival recession that may expose the veneers [4].

\section{Dietary Modifications}

On contrary to natural dentitions, Porcelain veneers resist stains such as coffee, tea and cigarettes smoke because they are manufactured using high-tech materials $[2,3]$. Although, it's often advisable to avoid stain-causing food and beverages as they can become stained over time likewise natural dentitions. This includes coffee, tea, red wine, soya sauce, dark sodas, and berries are the few examples that may cause tooth discoloration. Another common cause of tooth discoloration is smoking. Therefore, smoking cessation is advisable for patients with porcelain veneers [4].

\section{Special Consideration for Parafunctional Habits}

Nigh guard or occlusal guard is recommended for patients with parafunctional habits such as bruxism that is characterized as clenching or grinding of teeth during the night. Bruxism can induce a great amount of stress on the teeth, potentially cause damage to porcelain veneers as well as natural dentition. Customized Night guards may prevent porcelain veneers from damage, and it can last for a longer period. This device may reduce the stress on the teeth due to bruxism $[3,4]$.

\section{Avoid Chewing on Hard Objects}

Veneers are extremely durable. However, they are not considered as strong as natural dentitions. Therefore, patients should avoid chewing on very hard objects, such as pens, ice cubes, nails, etc. Hence, chewing on these or other hard objects might cause damage to porcelain veneers $[3,4]$.

\section{Maintenance and Follow-Up Visits}

After veneers have been placed, patients are advised to schedule a follow-up visit for polishing the veneers because porcelain veneers should be polished with a specially formulated, non-abrasive paste. In addition, regular check-ups after every six months and thorough cleaning and comprehensive examination are often recommended by the dentists. This will give a Cosmetic Dentist an opportunity to anticipate or sort any problems with the veneers and solve potential issues before it happens [1, 3 \& 4]

\section{Conclusion}

Therefore, it is mandatory for dental professionals to reinforce brief instructions and advise their patients to practice good oral hygiene care when porcelain veneers are placed. Nevertheless, schedule regular dental visits that may have a positive impact on long term clinical predictability of porcelain veneers.

\section{References}

1. Porcelain Veneers Maintenance of Porcelain Veneers. American Academy of Cosmetic Dentistry (AACD)

2. Dental health and Veneers (2017) WebMD Medical Reference reviewed by Michael Friedman.

3. Dental Veneers Care Tips. Smiles on Michigan.

4. A Wilkinson Dental (2015) How to care for Dental Veneers. 